\title{
Erratum: Computable form of the Born-Markov master equation for open multilevel quantum systems [Phys. Rev. A 99, 022118 (2019)]
}

\author{
Xian-Ting Liang
}

(Received 7 October 2019; published 7 November 2019)

DOI: 10.1103/PhysRevA.100.059901

The coefficients $\bar{D}$ and $\bar{f}$ in our original paper are incorrect. A corrected version of the derivation on the coefficients is given here. However, for two models in our original paper, the reduced density matrix, calculated with the corrected $\bar{D}$ and $\bar{f}$ is very much the same as the results in the original paper. So the evolution figures of the reduced density matrix do not need to be corrected, and they are not replotted here. And, none of the discussions and conclusions were affected by the errors.

We start with correcting the coefficient $\bar{f}$. The calculating strategy of $\bar{D}$ and $\bar{f}$ are given in Fig. 1 .

According to our original paper, $\bar{f}$ is a integral,

$$
\bar{f}=\frac{1}{2} \int_{0}^{\infty} d \tau \int_{-\infty}^{\infty} d \omega J(\omega) \operatorname{coth}\left(\frac{\beta \omega}{2}\right) \cos (\omega \tau) \sin (\Delta \tau) .
$$

In order to calculate the integral, we consider a contour integral,

$$
I_{2}=\oint F_{2}(z) d z
$$

with

$$
F_{2}(z)=\frac{1}{2} J(z) \operatorname{coth}\left(\frac{z \beta}{2}\right) e^{i z \tau}=\frac{z \eta \Omega}{2\left(z^{2}+\Omega^{2}\right)} \frac{e^{\beta z / 2}+e^{-\beta z / 2}}{e^{\beta z / 2}-e^{-\beta z / 2}} e^{i z \tau} .
$$

It is clear that $F_{2}(z)$ has simple poles $z=i \Omega$ and $\frac{i 2 n \pi}{\beta}(n=0-3, \ldots)$. The contour integral can be broken down into the sum of four integrals as

$$
\oint F_{2}(z) d z=\left(\int_{-\infty}^{\varepsilon_{-}}+\int_{\varepsilon_{+}}^{\infty}+\int_{R}-\int_{\varepsilon}\right) F_{2}(z) d z .
$$

According to Jordan's lemma, we have

$$
\lim _{R \rightarrow \infty} \int_{R} F_{2}(z) d z=0
$$

and

$$
\lim _{\varepsilon \rightarrow 0} \int_{\varepsilon} F_{2}(z) d z=0
$$

So, according to the residue theorem, we have

$$
\int_{-\infty}^{\infty} F_{2}(z) d z=\left(\int_{A}^{B}+\int_{C}^{D}\right) F_{2}(z) d z=\oint F_{2}(z) d z=2 \pi i\left(\operatorname{Res}_{z=i \Omega}\left[F_{2}(z)\right]+\sum_{n=1,2, \ldots} \underset{z=2 n \pi i / \beta}{\operatorname{Res}}\left[F_{2}(z)\right]\right) .
$$

For the simple pole $z=i \Omega$, we have

$$
\operatorname{Res}_{z=i \Omega}\left[F_{2}(z)\right]=\frac{\eta \Omega}{4} \frac{e^{i \beta \Omega / 2}+e^{-i \beta \Omega / 2}}{e^{i \beta \Omega / 2}-e^{-i \beta \Omega / 2}} e^{-\Omega \tau}=\frac{\eta \Omega}{4 i} \cot \left(\frac{\beta \Omega}{2}\right) e^{-\Omega \tau},
$$

and for the simple poles $z=i 2 n \pi / \beta(n=1,2, \ldots)$,

$$
\sum_{n=1,2, \ldots} \operatorname{Res}_{z=i 2 n \pi / \beta}\left[F_{2}(z)\right]=\left.\sum_{n=1,2, \ldots} \frac{\eta z \Omega}{2\left(z^{2}+\Omega^{2}\right)} \frac{e^{\beta z / 2}+e^{-\beta z / 2}}{\frac{\beta}{2}\left(e^{\beta z / 2}+e^{-\beta z / 2}\right)} e^{i \Omega z}\right|_{z=(i 2 n \pi) / \beta}=\sum_{n=1,2, \ldots} 2 n \pi \frac{i \eta \Omega}{\Omega^{2} \beta^{2}-4 \pi^{2} n^{2}} e^{-(2 n \pi \tau) / \beta} .
$$




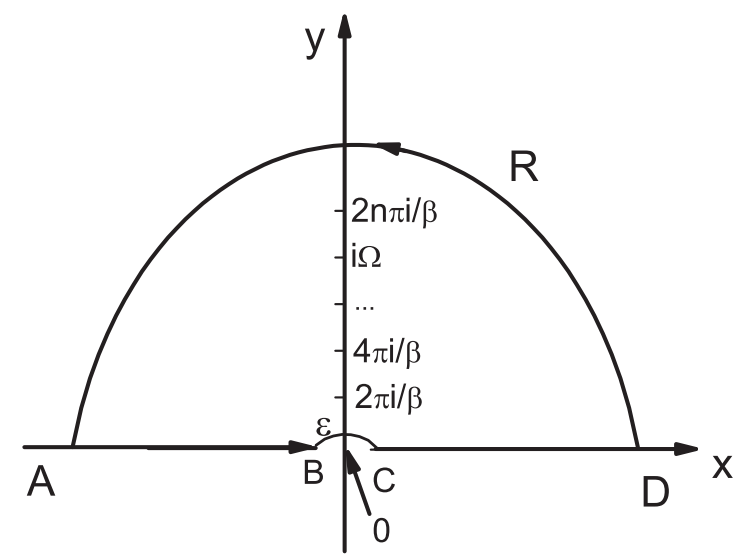

FIG. 1. The computing strategy of the coefficients $\bar{D}$ and $\bar{f}$.

Thus, when $\Omega \neq 2 k \pi / \beta k,[k \in(n=1,2, \ldots)]$, the contour integral can be written as

$$
\begin{aligned}
I_{2} & =2 \pi i\left[\operatorname{Res}_{z=i \Omega}\left[F_{2}(z)\right]+\operatorname{Res}_{z=-i 2 n \pi / \beta}\left[F_{2}(z)\right]\right] \\
& =2 \pi i\left[\frac{\eta \Omega}{4 i} \cot \left(\frac{\beta \Omega}{2}\right) e^{-\Omega \tau}+\sum_{n=1,2, \ldots} 2 n \pi \frac{i \eta \Omega}{\Omega^{2} \beta^{2}-4 \pi^{2} n^{2}} e^{-(2 n \pi \tau) / \beta}\right] \\
& =\frac{\pi \eta \Omega}{2} \cot \left(\frac{\beta \Omega}{2}\right) e^{-\Omega \tau}-\sum_{n=1,2, \ldots} 4 n \pi^{2} \frac{\eta \Omega}{\Omega^{2} \beta^{2}-4 \pi^{2} n^{2}} e^{-(2 n \pi \tau) / \beta} .
\end{aligned}
$$

When $\Omega=2 k \pi / \beta,[k \in(n=1,2, \ldots)]$, the simple pole $z=i \Omega$ and the simple pole $z=i 2 k \pi / \beta$ are the same point, it means that the pole becomes the second-order pole $z=i \Omega=i 2 k \pi / \beta$, thus, we can calculate the residues as [1]

$$
\operatorname{Res}_{z=i \Omega}\left[F_{2}(z)\right]=\lim _{z=i \Omega} \frac{d}{d z}\left[\frac{\eta \Omega z}{2(z+\Omega)^{2}}\left(z-\frac{i k 2 \pi}{\beta}\right)^{2} \operatorname{coth}\left(\frac{\beta z}{2}\right) e^{i z \tau}\right]=-\frac{i \eta}{4 \beta} e^{-\Omega \tau}+\frac{i \eta \Omega \tau}{2 \beta} e^{-\Omega \tau} .
$$

And, the contour integral can be written

$$
\begin{aligned}
I_{3} & =2 \pi i\left[\operatorname{Res}_{z=i \Omega}\left[F_{2}(z)\right]+\operatorname{Res}_{z=-i 2 n \pi / \beta}\left[F_{2}(z)\right]\right] \\
& =2 \pi i\left[-\frac{i \eta}{4 \beta} e^{-\Omega \tau}+\frac{i \eta \Omega \tau}{2 \beta} e^{-\Omega \tau}+\sum_{n=1,2, \ldots}^{n \neq k} 2 n \pi \frac{i \eta \Omega}{\Omega^{2} \beta^{2}-4 \pi^{2} n^{2}} e^{-(2 n \pi \tau) / \beta}\right] \\
& =\frac{\eta \pi}{2 \beta}(1-2 \Omega \tau) e^{-\Omega \tau}-\sum_{n=1,2, \ldots}^{n \neq k} 4 n \pi^{2} \frac{\eta \Omega}{\Omega^{2} \beta^{2}-4 \pi^{2} n^{2}} e^{-(2 n \pi \tau) / \beta} .
\end{aligned}
$$

So, when $\Omega \neq 2 k \pi / \beta,[k \in(n=1,2, \ldots)]$, we have

$$
\bar{f}=\int_{0}^{\infty} \operatorname{Re}\left(I_{2}\right) \sin (\Delta \tau) d \tau=\frac{\pi \eta \Omega \Delta}{2\left(\Omega^{2}+\Delta^{2}\right)} \cot \left(\frac{\beta \Omega}{2}\right)-\sum_{n=1,2, \ldots} \frac{\bar{\omega}_{n}^{2} \eta \Omega \Delta}{n\left(\Omega^{2}-\bar{\omega}_{n}^{2}\right)\left(\Delta^{2}+\bar{\omega}_{n}^{2}\right)},
$$

here $\bar{\omega}_{n}=2 n \pi / \beta$. When $\Omega=2 k \pi / \beta,[k \in(n=1,2, \ldots)]$, we have

$$
\bar{f}=\int_{0}^{\infty} \operatorname{Re}\left(I_{3}\right) \sin (\Delta \tau) d \tau=\frac{\eta \pi}{2 \beta} \frac{\Delta^{3}-3 \Delta \Omega^{2}}{\Delta^{2}+\Omega^{2}}-\sum_{n=1,2, \ldots}^{n \neq k} \frac{\bar{\omega}_{n}^{2} \eta \Omega \Delta}{n\left(\Omega^{2}-\bar{\omega}_{n}^{2}\right)\left(\Delta^{2}+\bar{\omega}_{n}^{2}\right)} .
$$

Next, we consider the convergence of this series. Assuming $\Omega \gg \Delta$, we have

$$
\sum_{n} \frac{\bar{\omega}_{n}^{2} \eta \Omega \Delta}{n\left(\Omega^{2}-\bar{\omega}_{n}^{2}\right)\left(\Delta^{2}+\bar{\omega}_{n}^{2}\right)}<\sum_{n} \frac{\bar{\omega}_{n}^{2}}{n} \frac{\eta \Omega \Delta}{\bar{\omega}_{n}^{4}+\left(\Omega^{2}-\Delta^{2}\right) \bar{\omega}_{n}^{2}}=\sum_{n} \frac{1}{n} \frac{\eta \Omega \Delta}{\bar{\omega}_{n}^{2}+\left(\Omega^{2}-\Delta^{2}\right)}<\sum_{n} \frac{1}{n} \frac{\eta \Omega \Delta}{\bar{\omega}_{n}^{2}}=\frac{\eta \Omega \Delta \beta^{2}}{4 \pi^{2}} \zeta(3) .
$$

Here, $\zeta$ (3) is a Riemann function [2], and it is convergent. We can approximately calculate the value of the series in $n^{3} \gg \frac{\eta \Omega \Delta \beta^{2}}{4 \pi^{2}}$. Namely, we can take any number $n \gg\left(\frac{\eta \Omega \Delta \beta^{2}}{4 \pi^{2}}\right)^{1 / 3}$ in the calculations. 
Finally, we correct the coefficient $\bar{D}$ :

$$
\bar{D}=\int_{0}^{\infty} d \tau \int_{0}^{\infty} d \omega J(\omega) \operatorname{coth}\left(\frac{\beta \omega}{2}\right) \cos (\omega \tau) \cos (\Delta \tau)
$$

When $\Delta \neq 0$, we have

$$
\bar{D}=\frac{\pi}{2} J(\Delta) \operatorname{coth}\left(\frac{\beta \Delta}{2}\right),
$$

and when $\Delta=0$ and $\Omega \neq 2 k \pi / \beta,[k \in(n=1,2, \ldots)]$, we have

$$
\begin{aligned}
\bar{D} & =\frac{1}{2} \int_{0}^{\infty} d \tau \int_{-\infty}^{\infty} d \omega J(\omega) \operatorname{coth}\left(\frac{\beta \omega}{2}\right) \cos (\omega \tau)=\int_{0}^{\infty} \operatorname{Re}\left(I_{2}\right) d \tau \\
& =\int_{0}^{\infty} d \tau\left[\frac{\pi \eta \Omega}{2} \cot \left(\frac{\beta \Omega}{2}\right) e^{-\Omega \tau}-\sum_{n=1,2, \ldots} 4 n \pi^{2} \frac{\eta \Omega}{\Omega^{2} \beta^{2}-4 \pi^{2} n^{2}} e^{-(2 n \pi \tau) / \beta}\right]=\frac{\pi \eta}{2} \cot \left(\frac{\beta \Omega}{2}\right)-\sum_{n=1,2, \ldots} \frac{\eta \Omega \bar{\omega}_{1}}{\left(\Omega^{2}-\bar{\omega}_{n}^{2}\right)} .
\end{aligned}
$$

When $\Delta=0$, and $\Omega=2 k \pi / \beta,[k \in(n=1,2, \ldots)]$, we have

$$
\bar{D}=\int_{0}^{\infty} \operatorname{Re}\left(I_{3}\right) d \tau=\int_{0}^{\infty} d \tau\left[\frac{\eta \pi}{2 \beta}(1-2 \Omega \tau) e^{-\Omega \tau}-\sum_{n=1,2, \ldots}^{n \neq k} 4 n \pi^{2} \frac{\eta \Omega}{\Omega^{2} \beta^{2}-4 \pi^{2} n^{2}} e^{-(2 n \pi \tau / \beta)}\right]=-\frac{\eta \pi}{2 \beta \Omega}-\sum_{n=1,2, \ldots}^{n \neq k} \frac{\eta \Omega \bar{\omega}_{1}}{\left(\Omega^{2}-\bar{\omega}_{n}^{2}\right)} .
$$

Now, we consider the convergence of this series,

$$
\sum_{n=1,2, \ldots} \frac{\eta \Omega \bar{\omega}_{1}}{\left(\Omega^{2}-\bar{\omega}_{n}^{2}\right)}<\sum_{n=1,2, \ldots} \frac{\eta \Omega \bar{\omega}_{1}}{-\bar{\omega}_{n}^{2}}=-\frac{\eta \Omega \bar{\omega}_{1} \beta^{2}}{4 \pi^{2}} \zeta(2) .
$$

Here, $\zeta(2)$ is the Riemann function, and it is convergent. We can approximately calculate the value of the series in $n^{2} \gg \frac{\eta \Omega \bar{\omega}_{1} \beta^{2}}{4 \pi^{2}}=$ $\eta \Omega \beta / 2 \pi$. Namely, we can take any number $n \gg \sqrt{\eta \Omega \beta / 2 \pi}$ in the calculations.

I would like to thank my master student Mr. Chang-Yao Liao for his helpful discussion. This project was sponsored by the National Natural Science Foundation of China (Grant No. 21773131) and the K. C. Wong Magna Foundation of Ningbo University.

[1] C. L. Siegel, Analytic Functions of Several Complex Variables (Kendrick Press, Heber City, UT, 1950), Vol. 75, pp. $29-33$.

[2] M. R. Bicknell, Math. Mag. 40, 275 (1967). 